\title{
Análisis determinístico de la red de regulación génica involucrada en la expresión y función del factor de transcripción $\sigma^{32}$ en Escherichia coli
}

\author{
Diana Carolina Clavijo Buriticá 1,2, Bárbara Vêleria Mejía , Lina María Rojas ${ }^{3}$, Héctor Leandro Sáenz Castro ${ }^{2}$ \& Juvenal Yosa Reyes ${ }^{4}$
}

1 Departamento de Biología, Universidad Nacional de Colombia. Bogotá, Colombia.

2 Facultad de Medicina, Fundación Universitaria Juan N. Corpas. Bogotá, Colombia.

3 Departamento de Ingeniería Quimica, Universidad de los Andes. Bogotá, Colombia.

4 Departamento de Ciencias Básica, Universidad Simón Bolívar. Barranquilla, Colombia

\begin{abstract}
Motivation: The expression of $\sigma^{32}$ factor is a response of E. coli under thermal shock conditions. The mechanism of induction of chaperone proteins and thermal shock proteases has been well studied, but much of the regulation network of the $\sigma^{32}$ factor is not fully understood. For this reason, in this work a deterministic model of the network is proposed, which was solved by means of the tools present in Matlab ${ }^{\circledR}$ and then evaluated the influence of the transcription, translation and degradation speed on all concentrations of the species involved in the network, in order to determine which of these factors is the one that the cell possibly employs to regulate the expression of the factor $\sigma^{32}$.

Results: In the resolution of the ordinary equation system using the ode 45 algorithm, it was corroborated that the mean life time of sigma 32 is 1 minute for the concentration in steady state. In addition, variations in rpoH transcription constants and $\mathrm{o}^{32} \mathrm{mRNA}$ degradation in the network $\left(k_{1}, k_{2}\right)$ were found to be non-significant in $\sigma^{32}$ factor production, nor in the production of thermal shock proteins. On the other hand, from sensitivity analysis it was inferred that the $k_{3}$ translation constant of $\sigma^{32} \mathrm{RNA}$ is the most critical speed for regulating the expression of the $\sigma^{32}$ factor in the network. Finally, with respect to $k_{12}$ it is concluded that it is an important term in the stabilization of the $\sigma^{32}$ factor.
\end{abstract}

Availability and Implementation: The analysis was conducted at MatLab_R 2011a and the source code is found in the Supplemental Material.

Keywords: Deterministic model, Transcription factor, $\sigma^{32}$ factor, Gene regulation network

Autor de correspondencia:

Diana Clavijo

diana.calvijo@juanncorpas.edu.co

Como citar: Clavijo DC, Mejía BV, Rojas LM, Sáenz HL, Reyes JY. Análisis determinístico de la red de regulación génica involucrada en la expresión y la función del factor de transcripción $\sigma 32$ en Escherichia coli. Revista Cuarzo 2017; 23 (2) 7-15.

Recibido: 9 de agosto de 2017 Aceptado: 20 de octubre de 2017 Publicado: 30 de diciembre de 2017 


\section{Introducción}

La inducción de las proteínas de choque térmico en Escherichia coli es principalmente causado por el aumento en concentración del factor $\sigma^{32}$ que se expresa del gen rpoH. El incremento de los niveles de $\sigma^{32}$ depende tanto de la activación de la síntesis como de la degradacion de la proteína (1-3).

Esta proteína es un factor muy inestable, con una vida media que va de 1 a 4 minutos y que disminuye a medida que aumenta la temperaturura (4), está presente dentro de la célula con un nivel basal de algunas moléculas en estado libre. Es responsable de la producción de un gran número de proteínas chaperonas y proteasas necesarias para la regulación de la fisiología de la célula.

Un complejo de $\sigma^{32}$ y RNA polimerasa denominado como holoenzima $\sigma^{32}\left(\mathrm{E}^{32}\right)$ reconoce los genes de estas proteínas chape-ronas (Jcompgene y FtsHgene), en seguida ésta holoenzima se une a la región del promotor induciendo la transcripción, traduc-ción y por ende la expresión de algunas proteínas de choque tér-mico (chaperonas) como DnaK, DnaJ y GrpE, entre otras $(5,6)$. Estas proteínas chaperonas inducen la formación del complejo JKE (Jcomp), el cual a su vez se une al factor $\sigma^{32}$ para producir el complejo Jcomp- $\sigma^{32}$. Éste último junto con la proteína de cho-que FtsH promueven la degradación del factor $\sigma^{32}$ y la regenera-ción del complejo JKE (Figura 1).

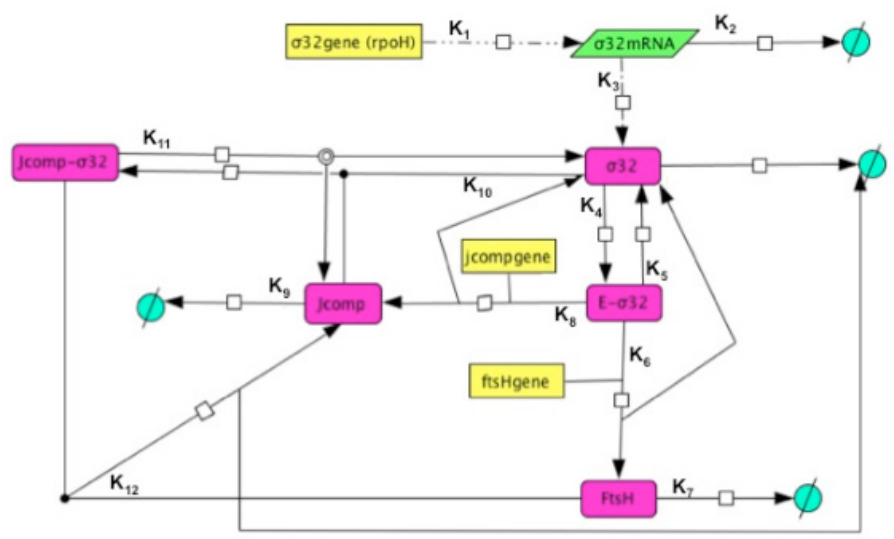

Figura I. Red de regulación del factor- $\sigma^{32}$. Después de que la proteína $\sigma^{32}$ es sintetizada, esta ayuda en la expresión de proteínas de respuesta al choque térmico como las proteasas y chaperonas. En amarillo se representa el DNA; en verde el RNA, en magenta las proteinas. La figura se encuentra construida sobre notación estándar SBGN (Systems Biology Graphical Notation).

Es así como la regulación del Factor $\sigma^{32}$ es un proceso multivalente, que considera no sólo la transcripción y traducción del gen rpoH sino tambien los controles postraduccionales que se encuentran involucrados (7).

Varios estdudios como los realizados por Kamath-Loeb y Gross en 1991 (8), Nagai y colaboradores en 1991 (9) y Morita y colaboradores en 1999 (1), indican que la abundancia celular del Factor $\sigma^{32}$ se encuentra regulada a nivel traduccional a través de la estructura secundaria del mRNA $\left(\sigma^{32} \mathrm{mRNA}\right)$. Dos secuencias en el mRNA de rpoH, una cerca del sitio de inicio de la traducción y otra interna, forman un elemento de estructura secundaria que es inhibido por altas temperaturas, permitiendo la traducción. Recientemente en 2013 Lim y colaboradores (10) indican como resultados de sus estudios que el Factor $\sigma^{32}$, se regula después de la traducción a través de procesos de degradación y por la inhibición directa de su actividad y que debe estar asociada con la membrana interna para ser regulada adecuadamente.

En esta investigación se desarrolla un modelo determinístico para representar la regulación de la proteína $\sigma^{32}$ como respuesta de $E$. coli ante el choque térmico y posteriormente se evalua el efecto de la velocidad de transcripción de $\mathrm{rpoH}$, la traducción y degradación de $\sigma^{32} \mathrm{mRNA}$ sobre la dinámica de la red para finalmente determinar cual de estos factores es el que la célula posiblemente emplea para regular la expresión del Factor $\sigma^{32}$.

Se presenta un análisis de sensibilidad indicando el rol de la síntesis de $\sigma^{32}$ en su regulación: partiendo del supuesto de que la inducción y la declinación de las proteínas de choque térmico pueden ser controladas cambiando la velocidad de transcripción de rpoH $\left(k_{1}\right)$, traducción $\left(k_{3}\right)$ y degradación $\left(k_{2}\right)$ de $\sigma^{32}$ mRNA del factor $\sigma^{32}(11)$.

\section{Métodos}

Tomando como base el modelo biológico de la red de regulación del factor $\sigma^{32}$ (Figura 1), las constantes de velocidad $\mathrm{k}$ para cada una de las reacciones implicadas en la red (Tabla 1) y las condiciones iniciales de concentración para cada especie de la red (Tabla 2), se planteó el sistema de ecuaciones diferenciales que representan la interacciones entre las especies (Ver ecuaciones 1 a 6).

$$
\begin{aligned}
& \frac{\left(d \sigma^{32} m R N A\right)}{d t}=k_{1}\left[\sigma \_g e n^{32}\right]-k_{2}\left[\sigma^{32} m R N A\right]-k_{3}\left[\sigma^{32} m R N A\right] \\
& \frac{d \sigma^{32}}{d t}=k_{3}\left[\sigma^{32} m R N A\right]+k_{s}\left[E_{-}-\sigma^{32}\right] \\
& +k_{8}\left[E_{-}-\sigma^{32}\right][j \text { compgen] } \\
& -k_{4}\left[\sigma^{32}\right]+k_{11}\left[J \text { comp }-\sigma^{32}\right] \\
& -k 10\left[\sigma^{32}\right][\text { Jcomp }] \\
& +k_{6}\left[\text { ftshgen] [E_ }\left(\sigma^{32}\right)\right] \\
& -k_{12}\left[\text { Jcomp_- } \sigma^{32}\right][\mathrm{FtsH}] \\
& \begin{array}{c}
\frac{\left(d E_{-}-\sigma^{32}\right)}{d t}=k_{4}\left[\sigma^{32}\right]-k_{s}\left[E_{-}-\sigma^{32}\right]-k_{\sigma}\left[E_{-}-\sigma^{32}\right][\text { ftshgen }] \\
-k_{s}\left[E_{-}-\sigma^{32}\right][j \text { compgen }]
\end{array} \\
& \frac{d F t s H}{d t}=\frac{k_{6}\left[E_{-}-\sigma^{32}\right][f t s h g e n]-k_{7}[\text { Fts } H]}{-k_{12}[\text { Fts } H]\left[\text { Jcomp }-\sigma^{32}\right]} \\
& \frac{d J_{\text {comp }}}{d t}=k_{11}\left[J_{c o m p}-\sigma^{32}\right]+k_{8}\left[E_{-}-\sigma^{32}\right][j \text { compgen }] \\
& +k_{12}[\text { FtsH }]\left[J \text { comp_- } \sigma^{32}\right] \\
& -k_{10}\left[\text { Jcomp] } \sigma^{32}\right]-k_{9}[J \text { comp] } \\
& \frac{\left(d J_{c o m p}-\sigma^{32}\right)}{d t}=k_{10}\left[\sigma^{32}\right]\left[J_{c o m p}\right]-k 11\left[J_{c o m p}-\sigma^{32}\right] \\
& -k_{12}\left[\text { Jcomp_- } \sigma^{32}\right][\mathrm{FtsH}]
\end{aligned}
$$


Para este modelo se asumió una cinética relativa a las ecuaciones de velocidad para las reacciones elementales que describen el sistema, bajo los parámetros que asume la ley de acción de masas para todas las especies de la red en equilibrio dinámico. El balance de materia que considera la diferencia entre las reacciones de generación o producción de la especie y las reacciones de consumo de la misma en la red fue considerado para todas las especies bajo los siguientes supuestos:

* Depreciación del factor de dilución de la célula.

* Concentración suficiente y disponible de nucleótidos a nivel celular.

* Para la especie E- $\sigma^{32}$, pese a ser una proteína que actúa como un factor de transcripción para la expresión algunas proteínas chaperonas, que actúa en un proceso reversible de unión y separación del promotor del gen y que considera velocidades de unión y separación muy pequeñas y proporcionales, en este modelo no se asume la reversibilidad mencionada para la inducción de los genes jcompgen y ftshgen, tan solo se asume una ireversibilidad para las reacciones de unión de este factor de transcripción al gen, que son controladas por $k_{6} \mathrm{y} k_{8}$ (no se cuenta con los valores experimentales de la velocidad de separación) asumiendo también una diferencia significativa en los valores de las velocidades de unión y separación para cada caso, por lo anterior estas reacciones fueron asumidas como reacciones de consumo de la especie E- $\sigma_{32}$ para el balance de masa.

Posterior al planteamiento matemático del sistema, fueron obtenidos los perfiles de concentración mediante la función ode45 para solución numérica de ecuaciones diferenciales, implementada en Matlab.

Tabla I. Constantes de velocidad para la red de regulación de $\sigma^{32}$.

$\begin{array}{cccc}\begin{array}{c}\text { Constante } \\ (\mathrm{k})\end{array} & \text { Valor de } k & \begin{array}{c}\text { Constante } \\ (\mathrm{k})\end{array} & \text { Valor de } k \\ k_{1} & 1.4 \times 10^{-5} \mathrm{~s}^{-1} & k_{7} & 7.40 \times 10^{-11} \mathrm{~s}^{-1} \\ k_{2} & 1.4 \times 10^{-6} \mathrm{~s}^{-1} & k_{8} & 4.41 \times 10^{-3}(\mathrm{~nm} * \mathrm{~s})^{-1} \\ k_{3} & 0.007 \mathrm{~s}^{-1} & k_{9} & 6.40 \times 10^{-10} \mathrm{~s}^{-1} \\ k_{4} & 0.7 \mathrm{~s}^{-1} & k_{10} & 3.27 \times 10^{-4}\left(\mathrm{~nm}^{*} \mathrm{~s}\right)^{-1} \\ k_{5} & 0.13\left(\mathrm{~nm} \mathrm{~s}^{-1}\right. & k_{11} & 4.4 \times 10^{-4} \mathrm{~s}^{-1} \\ k_{6} & 4.41 \times 10^{-3}\left(\mathrm{~nm} \mathrm{~s}^{-1}\right. & k_{12} & 1.28 \times 10^{-6}\left(\mathrm{~nm}^{*} \mathrm{~s}\right)^{-1}\end{array}$

Tabla 2. Concentraciones inciales en la red de regulación de $\sigma^{32}$.

\begin{tabular}{cccc}
$\begin{array}{c}\text { Especie } \\
\text { (Componente) }\end{array}$ & $\begin{array}{c}\text { Concentración } \\
\text { inicial }(\mathrm{nm})\end{array}$ & $\begin{array}{c}\text { Especie } \\
\text { (Componente) }\end{array}$ & $\begin{array}{c}\text { Concentración } \\
\text { inicial }(\mathrm{nm})\end{array}$ \\
\hline$\sigma^{32}$ gen & 1 & Jcompgen & 1 \\
$\sigma^{32}$ mRNA & 6 & Jcomp & 56 \\
$\sigma^{32}$ & 9 & Jcomp- $\sigma^{32}$ & 7 \\
ftshgen & 1 & $E-\sigma^{32}$ & 1 \\
FtsH & 93 & &
\end{tabular}

Las gráficas de sensibilidad para las concentraciones de metabol itos, se obtuvieron mediante un loop en Matlab para 5 iteraciones en los cuales cada constante a evaluar se multiplicaba cada vez por un factor de 10 . De esta manera, para $k_{1}$ las variaciones fueron: $1.4 \times 10^{-7}-1.4 \times 10^{-6}-1.4 \times 10^{-5}-1.4 \times 10^{-4}-1.4 \times 10^{-3}$, por ejemplo.

Para complementar el análisis de sensibilidad se realizaron pruebas de knockout en las que se realizó la simulación llevando a cero las constantes de transcripción, traducción y degradación y las concentraciones de las especies de las proteínas chaperonas (escenarios de simulación).

Tabla 3. Escenarios de Simulación. X representa un valor de cero en la constante y en la concentración de la proteína correspondiente. Sólo se muestran los datos para los escenarios $1,2,3$ y 12 .

\begin{tabular}{ccccc} 
Variable & 1 & 2 & 3 & 12 \\
\hline$k 1$ & $x$ & & & \\
$k 2$ & & $x$ & & \\
$k 3$ & & & $x$ & $x$ \\
Fts $H$ & & & & $x$ \\
Jcomp & & & & $x$
\end{tabular}

\section{Algoritmo}

El algoritmo empleado en este trabajo se muestra en el material complemetario.

\section{Resultados}

Después de planteado el modelo deterministico de la red de regulación génica involucrada en la expresión y función del factor de transcripción $\sigma^{32}$ en $E$. coli, se realizó la simulación para todo el sistema en un tiempo $\mathrm{t}=1 \mathrm{~min}$, teniendo en cuenta la vida media de la proteína $\sigma^{32}$ (Figura 2).

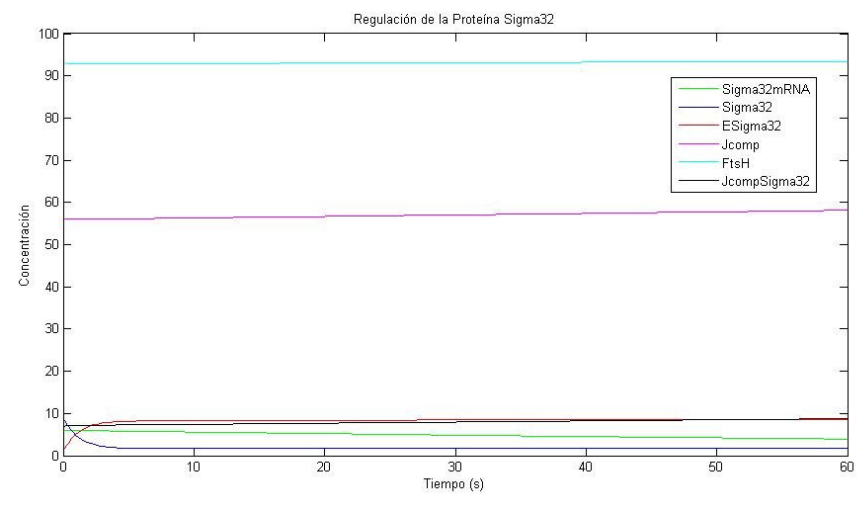

Figura 2. Simulación en un tiempo $t=1 \mathrm{~min}$, de la red de regulación génica involucrada en la expresión y función del factor de transcripción $\sigma^{32}$ en E. coli. Modelo determinístico implementado en MatLab. Las líneas representan con un color particular las diferentes especies químicas involucradas en el modelo según la convención en el recuadro superior derecho. Se representan sobre las abscisas la variación en el tiempo y sobre las ordenadas la variación de la concentración. 
Posteriormente se realizaron varias simulaciones a diferentes tiempos $\mathrm{t}$ con un máximo de $\mathrm{t}=5 \mathrm{~h}$, con el fin de encontrar el tiempo $t$ en el cual el sistema alcanza un estado estacionario (Figura 3).

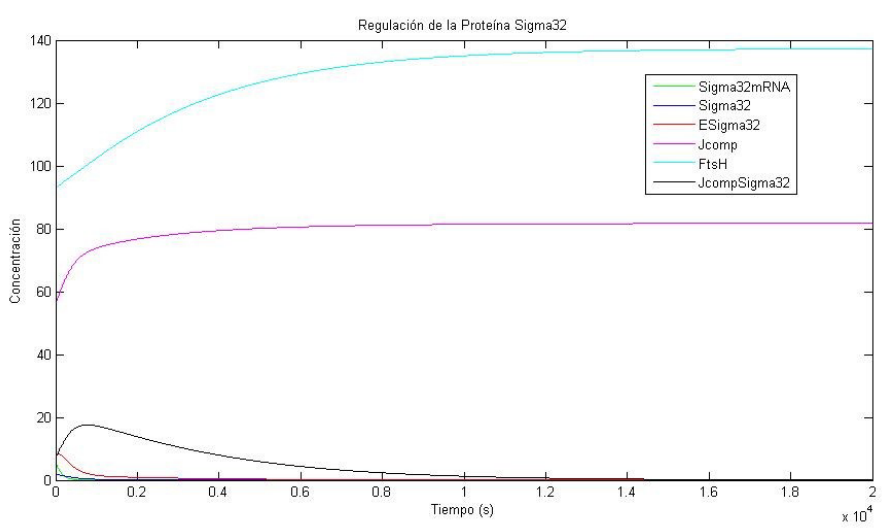

Figura 3. Simulación en un tiempo $t=5 \mathrm{~h}$, de la red de regulación génica involucrada en la expresión y función del factor de transcripción $\sigma^{32}$ en E. coli. Modelo determinístico implementado en MatLab. Se nota la diferencia de comportamiento de las especies respecto de la Figura1.

Con el fin de obtener mayor precisión y asegurar que el modelo planteado funcionaba adecuadamente, se modeló la red con el Sofware CellDesigner $4.3(12,13)$ y se realizaron las simulaciones con COPASI (14) obteniendo resultados similares (Figura 4).

Se hizo un análisis de estabilidad del sistema variando los valores de las constantes cinéticas más representativas: $k_{1}$ que es la encargada de regular la velocidad de transcripción del gen $\mathrm{rpoH}$ ( $\sigma^{32}$ gene), $k_{2}$ que regula la tasa de cambio de la degradación espontánea del mRNA ( $\left.\sigma^{32} \mathrm{mRNA}\right)$ y $k_{3}$ que regula la velocidad de síntesis de la proteína $\sigma^{32}$.

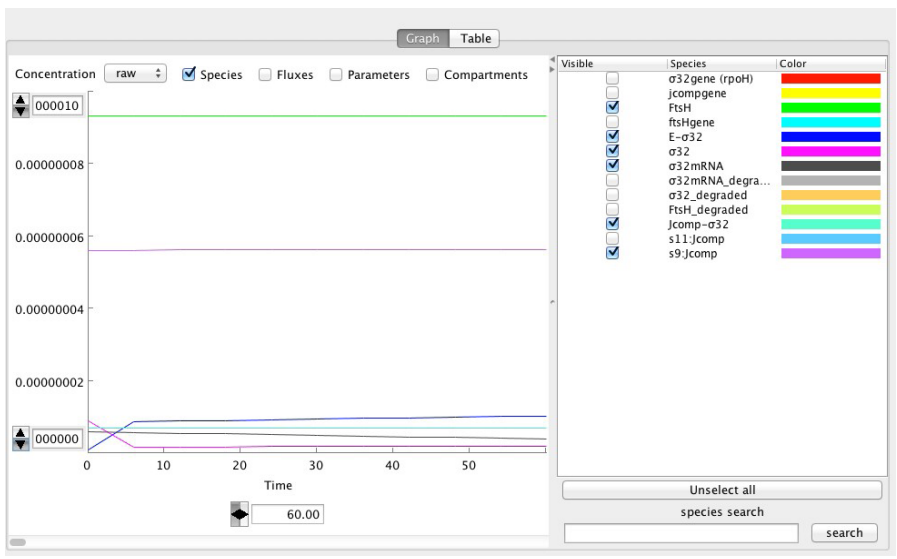

Figura 4. Simulación en un tiempo $\mathrm{t}=1 \mathrm{~min}$, de la red de regulación génica involucrada en la expresión y función del factor de transcripción $\sigma^{32}$ en E. coli. Modelo determinístico implementado en CellDesigner 4.3. Se presenta la interface del programa en la cual en el recuadro derecho se seleccionan las especies que se quieren visualizar con su respectiva notación de colores. Esta figura presenta los mismos resultados que la Figura1 obtenida con Matlab.

Cada uno de los valores de $k$ se variaron como se indica en la metodología para evaluar el efecto que éstos tienen sobre la ex- presión dinámica $\left(1 / \mathrm{nm}^{*} \mathrm{~s}\right)$ de las moléculas más representativas de la red como $\sigma^{32}$ mRNA, $\sigma^{32}$, E- $\sigma^{32}$, FtsH, Jcomp y Jcomp- $\sigma^{32}$. (Figuras 5, 6 y 7).
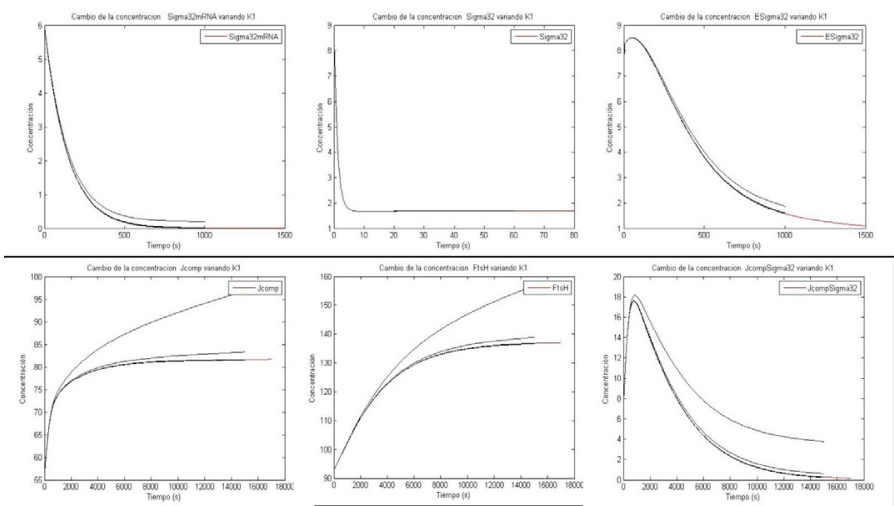

Figura 5. Cambio de la concentración de las especies de la red (en la fila superior de izquierda a derecha: $\sigma^{32} \mathrm{mRNA}, \sigma^{32}, \mathrm{E}-\sigma^{32}$, en la inferior, de izquierda a derecha Jcomp, FtsH, Jcomp y Jcomp- $\sigma^{32}$ ) con respecto a las variones en los valores de la tasa de transcripción de rpoH $\left(k_{1}\right)$. Modelo deterministico implementado en MatLab.
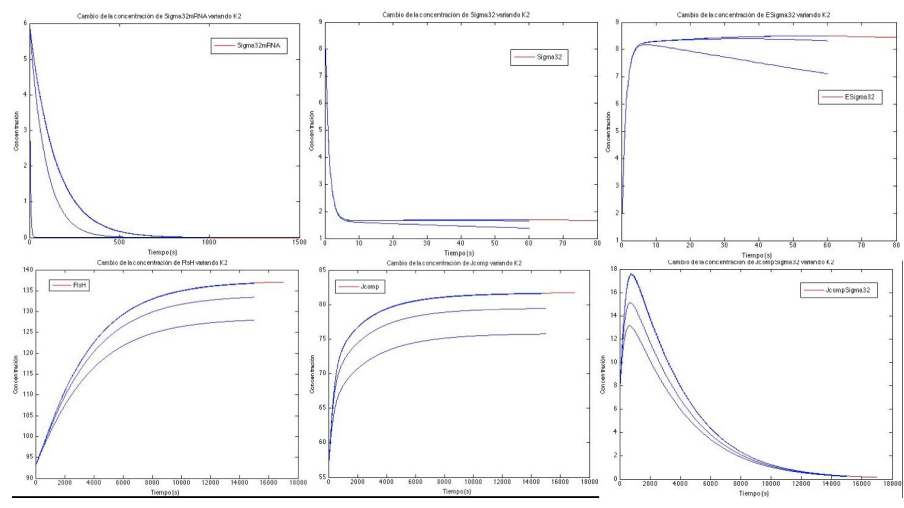

Figura 6. Cambio de la concentración de las especies de la red (en la fila superior: $\sigma^{32}, \mathrm{E}-\sigma^{32}$, en la fila inferior: FtsH, Jcomp y Jcomp- $\sigma^{32}$ ), con respecto a las variones en los valores de la tasa de degradación de $\sigma^{32}$ mRNA $\left(k_{2}\right)$. Modelo deterministico implementado en MatLab.

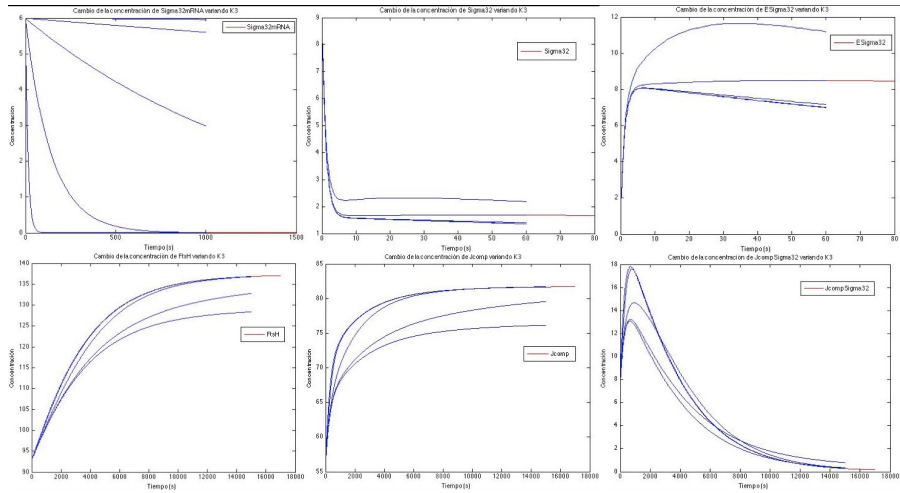

Figura 7. Cambio de la concentración de las especies de la red (en la fila superior: $\sigma^{32}, \mathrm{E}-\sigma^{32}$, en la fila inferior: FtsH, Jcomp y Jcomp- $\sigma^{32}$ ), con respecto a las variones en los valores de la tasa de traducción de $\sigma^{32}$ mRNA $\left(k_{3}\right)$. Modelo deterministico implementado en MatLab. 
Adicionalmente se evaluó el efecto de $k_{12}$ sobre el sistema debido a que está involucrada en la reacción de degradación de $\sigma^{32}$ y que a su vez incrementa la concentración de Jcomp. (Figura 8).
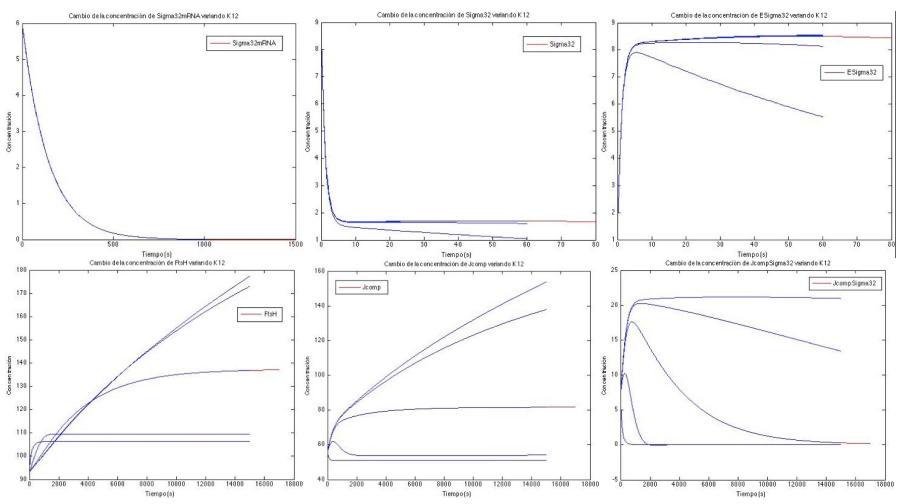

Figura 8. Cambio de la concentración de las especies de la red (en la fila superior: $\sigma^{32}, \mathrm{E}-\sigma^{32}$, en la fila inferior: FtsH, Jcomp y Jcomp- $\sigma^{32}$ ), con respecto a las variones en los valores de la tasa de descomposición del complejo Jcomp- $\sigma^{\wedge} 32$ y de inducción a la degradación de la proteína $\sigma^{32}\left(k_{12}\right)$. Modelo deterministico implementado en MatLab.

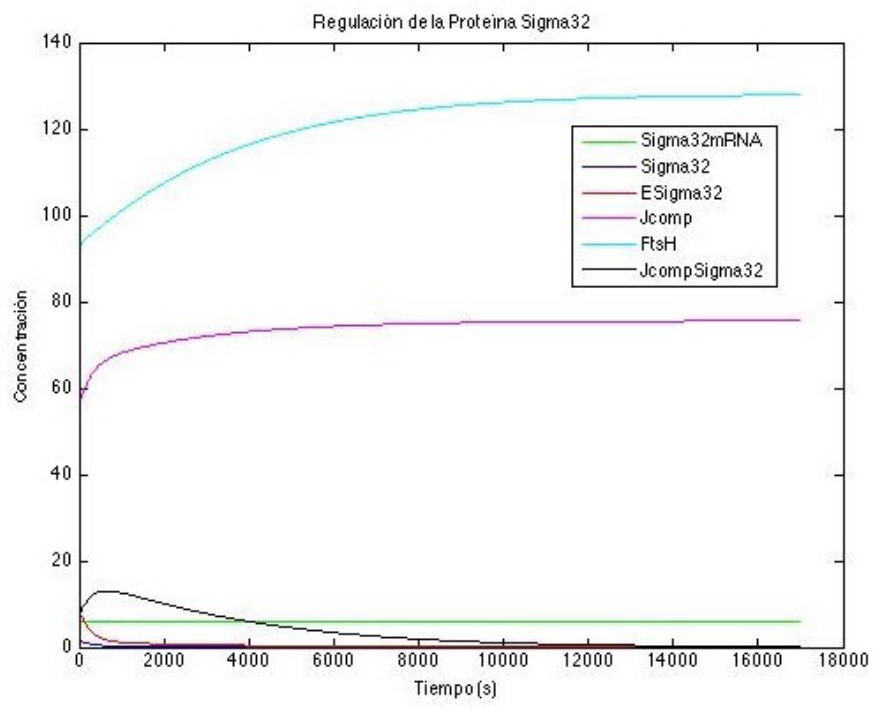

Figura 9. Simulación bajo las condiciones del escenario 3 de la red de regulación génica involucrada en la expresión y función del factor de transcripción $\sigma^{32}$ en E. coli. Modelo determinístico implementado en MatLab.

Dentro del análisis de sensibilidad se llevan las constantes $k_{1}, k_{2} y$ $k_{3}$ a cero para determinar cual factor (transcripción de rpoH, traducción o degradación de $\sigma^{32}$ ) es el que la célula usa para regular la dinámica de la red. Para complementar este análisis se dejaron las condiciones iniciales de las proteínas FtsH y Jcomp en cero. Con esto se generaron 12 escenarios de simulación (Tabla 3 ).

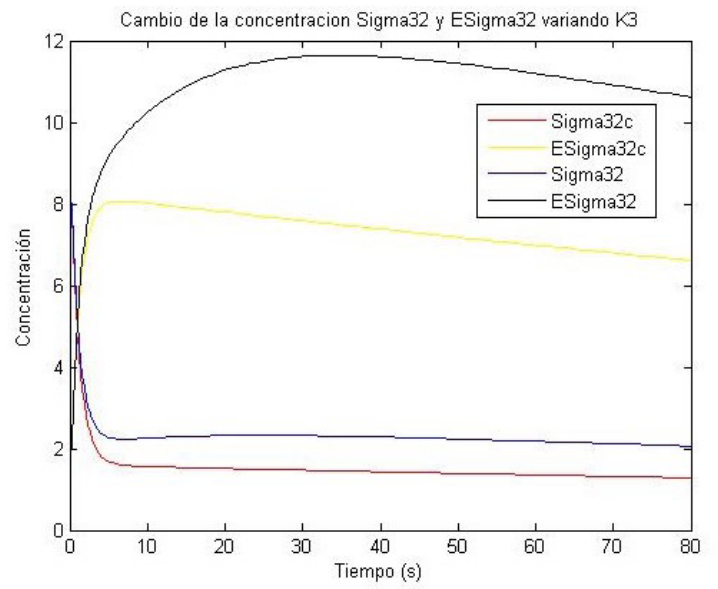

Figura 10. Simulación bajo las condiciones del escenario 3 de la red de regulación génica involucrada en la expresión y función del factor de transcripción $\sigma^{32}$ en $E$. coli. Los nombres que incluyen el subindice c, indican que son las gráficas para cuando $k_{3}$ es cero, mientras que las que no lo tiene son con el $k_{3}$ establecido.

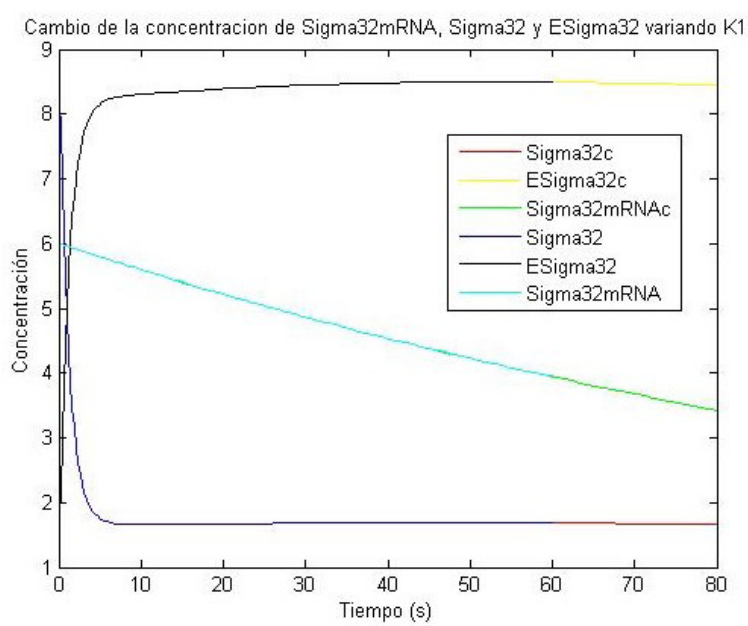

Figura ||. Simulación bajo las condiciones del escenario 1 de la red de regulación génica involucrada en la expresión y función del factor de transcripción $\sigma^{32}$ en E. coli. Los nombres que incluyen el subíndice c, indican que son las gráficas para cuando k1 es cero, mientras que la que no lo tienen son con el $k_{1}$ establecido.

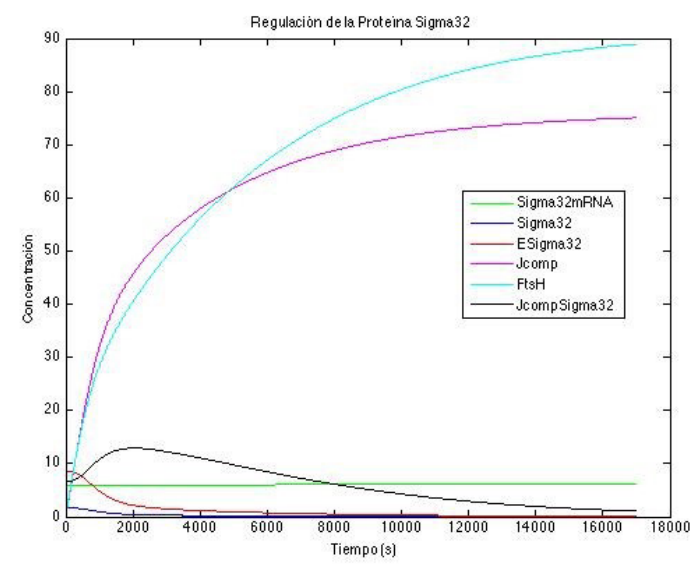

Figura 12. Simulación bajo las condiciones del escenario 12 de la red de regulación génica involucrada en la expresión y función del factor de transcripción $\sigma^{32}$ en E. coli. Modelo determinístico implementado en MatLab. 


\section{Discusión}

Partiendo de los resultados que se muestran en la Figura 2 se tiene que a excepción de las proteínas FtsH y Jcomp, las especies del sistema alcanzan un estado estacionario en los primeros segundos de funcionamiento de la red, por lo cual fue necesario ampliar el tiempo de simulación para corroborar la estabilidad de todas las especies involucradas y por ende del modelo completo. De acuerdo a los resultados de la simulación en un tiempo $\mathrm{t}=5 \mathrm{~h}$, de la red de regulación génica involucrada en la expresión y función del factor de transcripción $\sigma^{32}$ en E. coli. (Figura 3), se tiene que el modelo está bien planteado ya que cada una de las especies de la red consiguen llegar a un estado estacionario dentro de las primeras 4 horas planteadas en la simulación. Se considera que el comportamiento de FtsH y Jcomp, que son las especies que más tardan en llegar a un estado estacionario, posiblemente se debe a las altas concentraciones iniciales que se consideraron en el modelo, las cuales fueron necesarias para evitar una acumulación de la proteína $\sigma^{32}$ a lo largo de la simulación.

Los resultados anteriores fueron corroborados mediante el Sofware de uso libre CellDesigner 4.3 en el cual las simulaciones arrojaron resultados similares a los planteados en el modelo que se está evaluando (Figura 2 y Figura 4), lo que corrobora que el modelo determinístico planteado en este trabajo para la red de regulación génica involucrada en la expresión y función del factor de transcripción $\sigma^{32}$ en E. coli es coherente y muestra una buena aproximación. Además, éste sistema no es solamente coherente matemáticamente sino que también refleja el compor- tamiento biológico esperado para una red de regulación de este tipo, como por ejemplo el comportamiento inversamente proporcional que se muestra entre la proteína $\sigma^{32}$ y el complejo E- $\sigma^{32}$; éste último muestra un incremento en su concentración a medida que $\sigma^{32}$ se empieza a consumir dentro del sistema para ayudar a la formación de $\mathrm{E}-\sigma^{32}$.

$\sigma^{32}$ mRNA no presenta una variación significativa en el tiempo puesto que la velocidad de síntesis $\left(k_{1}=1.4 \times 10^{-5} \mathrm{~s}^{-1}\right)$ es mucho menor comparada con la velocidad de traducción $\left(k_{2}=7 \times 10^{-3}\right.$ $\left.\mathrm{s}^{-1}\right)$, lo que biológicamente indica que todo lo que se está transcribiendo de rpoH, se traduce casi de forma inmediata.

Por su parte Jcomp- $\sigma^{32}$ muestra una variación dinámica muy pequeña, lo cual es coherente con el comportamiento biológico de la red, puesto que hay una concentración muy elevada de la proteína FtsH en el medio celular, lo que implica que todo el complejo Jcomp- $\sigma^{32}$ que se genera se une a la proteína FtsH para degradar la proteína $\sigma^{32}$.

Al confirmar que el sistema matemático se ajusta al modelo biológico, se exploró el efecto de la velocidad de transcripción de rpoH $\left(k_{1}\right)$, la velocidad de traducción $\left(k_{3}\right)$ y de degradación $\left(k_{2}\right)$ de $\sigma^{32} \mathrm{mRNA}$ en la dinámica de la red con la finalidad de establecer cual de estas variables es la que posiblemente emplea la célula para regular la expresión del factor- $\sigma^{\wedge} 32$.

Así, como se mencionó en la metodología cada $\mathrm{k}$ fue evaluado con cinco puntos de variación sobre las especies del sistema, tal y como se indica en la Tabla 4.

Tabla 4. Efecto del cambio en los valores de $k_{1}, k_{2} \mathrm{y} k_{3}$, sobre la expresión dinámica ( $\left.1 / \mathrm{nm}^{*} \mathrm{~s}\right)$ de las moléculas más representativas de la red como $\sigma^{32} \mathrm{mRNA}, \sigma^{32}$, E- $\sigma^{32}$, FtsH, Jcomp y Jcomp- $\sigma^{32}$. (+ indica que con el aumento en el valor del k correspondiente la expresión del componente aumenta, = indica que con el aumento en el valor del $\mathrm{k}$ correspondiente la expresión del componente no presenta ningún cambio significativo y - indica que con el aumento en el valor del k correspondiente la expresión del componente disminuye).

\begin{tabular}{|c|c|c|c|c|c|c|}
\hline $\begin{array}{l}\text { Aumento en } \\
\text { Valores de k }\end{array}$ & $\mathrm{o}^{32} \mathrm{mRNA}$ & $0^{32}$ & $E-o^{32}$ & FtsH & Jcomp & Jcomp-o ${ }^{32}$ \\
\hline$k_{1}$ - Elevado & + & + & + & + & + & + \\
\hline$k_{2}$ - Elevado & $=$ & $=$ & $=$ & $=$ & $=$ & $=$ \\
\hline$k_{3}$ - Elevado & - & + & + & + & + & + \\
\hline
\end{tabular}

Al variar $k_{1}$ (Figura 5) se observa que la expresión de $\sigma^{32}$ en el sistema no cambia significativamente, sin embargo la concentración de $\sigma^{32} \mathrm{mRNA}$ muestra un aumento considerable, lo que indica que no todo el gen que se está transcribiendo se está traduciendo, puesto que para este escenario de simulación se mantiene el valor original de $k_{3}$ (Velocidad de traducción de $\sigma^{32} \mathrm{mRNA}$ ); estos resultados concuerdan con lo que biológicamente ocurre a nivel celular. Por otro lado el aumento que sufre la expresión de los demás componente de la red se puede explicar debido a las altas concentraciones consideradas en las condiciones iniciales de FtsH y Jcomp que se emplean en el modelo determinístico, ya que tan solo un pequeño aumento en la concentración de $\sigma^{32}$ es altamente sensible para la expresión de los complejos proteicos.

Con respecto al aumento de la velocidad de degradación de $\sigma^{32}$ mRNA ( $k_{2}$ elevado. Tabla 4), se observa que la expresión de $\sigma^{32}$ disminuye muy poco puesto que aunque se aumenta el valor de $\mathrm{k}$ éste sigue siendo muy bajo para afectar la síntesis del Factor $\sigma^{32}$ y por ende la expresión de los demás componente de la red de regulación (Figura 6).

Por otro lado, el efecto que presentan los cambios en la velocidad de traducción del $\sigma^{32}$ mRNA $\left(k_{3}\right)$ para el modelo determinístico planteado, también arrojan resultados biológicamente compatibles. Estos datos indican que con un aumento en la velocidad de traducción, la concentración de $\sigma^{32}$ mRNA diminuye y la expresión tanto de $\sigma^{32}$, como de E- $\sigma^{32}$, FtsH, Jcomp y Jcomp- $\sigma^{32}$ aumentan significativamente, tal y como se espera que ocurra a nivel celular (Figura 7 y Tabla 4), puesto que se requiere que para la expresión de una proteína la concentración de 
mRNA disminuya por la velocidad de traducción asociada. Con lo anterior se puede indicar que la velocidad de traducción es la que aparentemente emplea la célula para regular la expresión del Factor- $\sigma^{32}$, lo cual está de acuerdo con los resultados de los trabajos realizados por Kamath-Loeb y Gross en 1991 (8), Nagai y colaboradores en 1991 (9) y Morita y colaboradores en 1999 (1).

Adicional al análisis realizado anteriormente, en este trabajo se buscó evaluar en el modelo la reacción gobernada por $k_{12}$, en la que por acción de la interacción entre Jcomp- $\sigma^{32}$ y FtsH se consigue la degradación del factor- $\sigma^{32}$, obteniendo que un incremento tanto en el valor de $k_{12}$ como en las concentraciones de $\mathrm{E}-\sigma^{32}$, $\sigma^{32}$, FtsH, Jcomp y Jcomp- $\sigma^{32}$ disminuyen tal y como se presenta en la Figura 8. Este resultado era esperado, debido a que se está aumentando la velocidad de degradación de la proteína $\sigma^{32}$, empleando la proteína FtsH y el complejo Jcomp- $\sigma^{32}$. De esta manera se disminuye sus concentraciones y la concentración de E- $\sigma^{32}$ disponible en el medio según el mecanismo de regulación. Sin embargo, la constante $k_{12}$ no sólo implica la degradación de $\sigma^{32}$ sino el aumento de Jcomp en el medio, pero este disminuye ya que la velocidad de síntesis de Jcomp- $\sigma^{32}$ continúa siendo mayor comparada con la velocidad de producción de Jcomp.

Complementando el estudio con el análisis de sensibilidad que se planteó en la metodología, se obtuvo que llevando a cero k3 la concentración de FtsH, Jcomp- $\sigma^{32}$, E- $\sigma^{32}$ y Jcomp- $\sigma^{32}$ disminuyen debido a que no se está traduciendo $\sigma^{32} \mathrm{mRNA}$, afectando así todo el sistema (Figuras 9 y 10), lo cual concuerda con la propuesta que se realiza en cuanto a que es la velocidad de traducción la que aparentemente emplea la célula para regular la expresión del Factor- $\sigma^{32}$; mientras que cuando se lleva el valor de $k_{1}$ a cero (Figura 11), los valores en las concentraciones para la proteína $\sigma^{32}$ y E- $\sigma^{32}$ siguen el mismo comportamiento debido a que el valor de $k_{l}$ inicial es bajo $\left(k_{l}=1.4 \times 10^{-5} \mathrm{~s}^{-1}\right)$ y no existe un cambio significativo en el valor de $k_{1}$. Por otro lado, cuando el valor de $k_{2}$ es cero tampoco se presentan cambios representativos en los perfiles de concentración de $\sigma^{32}$ y E- $\sigma^{32}$, debido a que inicialmente este valor también es muy pequeño $\left(k_{l}=1.4 \times 10^{-6} \mathrm{~s}^{-1}\right)$. Además se puede observar (Figura 11) que $\sigma^{32} \mathrm{mRNA}$ disminuye debido a que la concentración inicial que hay en el medio que se va consumiendo en la síntesis de $\sigma^{32}$.

Para el escenario de simulación 12 (Figura 12) en donde $k_{3}$, Jcomp y FtsH se hacen cero se puede ver que la concentración del factor- $\sigma^{32}$ no llega a cero en 60 segundos, pues el mecanismo de degradación que supone la reacción entre Jcomp- $\sigma^{32}$ y FtsH no se lleva a cabo con la misma frecuencia, ya que no hay suficiente chaperona y proteasa. Sin embargo a medida que se produce $\sigma^{32}$ se obtiene Jcomp, FtsH y estos a la final estabilizan la producción del factor- $\sigma^{32}$ después de aproximadamente 4000 segundos. También cabe notar que las concentraciones de las proteínas de choque térmico llegan al estado estacionario en un dato menor comparado con la corrida de datos originales pues la concentración (suministro) solo proviene de la que se induzca por E- $\sigma^{32}$. Para terminar, la concentración de $\sigma^{32}$ mRNA permanece casi constante en la concentración inicial, pues al suponer $k_{3}=0$ no se consume para producir $\sigma^{32}$ pero se ve un poco el aumento por el proceso de transcripción.
A partir de estudio se recomienda continuar la investigación planteando un modelo en el que se considere para el balance de materia de la especie E- $\sigma^{32}$, la reversibilidad de las reacciones de inducción de los genes jcompgen (k-8) y ftshgen (k-6), siempre y cuando se cuente con los valores experimentales de las velocidades de separación del inductor transcripcional de cada gen, sin embargo con esto se esperaría que al ser bajas las velocidades de unión y separación y considerando una proporcionalidad entre ellas, en el modelo tengan que ser despreciadas ya que matemáticamente los términos se cancelarían.

\section{Conclusiones}

La velocidad de traducción de $\sigma^{32} \mathrm{mRNA}$ es la que posiblemente emplea la célula para regular la expresión del Factor- $\sigma^{32}$.

El factor $\sigma^{32}$ y las especies del sistema relacionadas con éste alcanzan un estado estacionario en los primeros 60 segundos, lo que corrobora los datos encontrados en la bibliografía para la vida media del factor.

Al multiplicar las constantes de transcripción y degradación por un factor de 100 y por un factor de (1/100) no se presentan cambios significativos en las concentraciones, por lo que se concluye que la célula no regula la producción del factor mediante estas reacciones.

Al multiplicar la constante de traducción por un factor de $100 \mathrm{y}$ por un factor de (1/100) se presentan cambios significativos en las concentraciones, por lo que se concluye que la célula regula la concentración de $\sigma^{32}$ mediante la traducción del $\sigma^{32} \mathrm{mRNA}$.

\section{Referencias}

1 Morita MT, Tanaka Y, Kodama TS, Kyogoku Y, Yanagi H, Yura T. Translational induction of heat shock transcription factor sigma32: evidence for a builtin RNA thermosensor. Genes Dev [Internet]. 1999 Mar 15; 13(6):655-65. Available from: http://www.ncbi.nlm.nih.gov/pubmed/10090722

2 Goff SA, Goldberg AL. Production of abnormal proteins in E. coli stimulates transcription of lon and other heat shock genes. Cell [Internet]. 1985 Jun; 41(2):587-95. Available from: http://www.ncbi.nlm.nih.gov/pub$\operatorname{med} / 3886165$

3 Straus DB, Walter WA, Gross CA. The heat shock response of E. coli is regulated by changes in the concentration of $\sigma 32$. Nature [Internet]. $1987 \mathrm{Sep}$ 24; 329(6137):348-51. Available from: http://www.ncbi.nlm.nih.gov/pub$\mathrm{med} / 3306410$

4 Morita MT, Kanemori M, Yanagi H, Yura T. Dynamic interplay between antagonistic pathways controlling the sigma 32 level in Escherichia coli. Proc Natl Acad Sci [Internet]. 2000 May 23; 97(11):5860-5. Available from: http://www.ncbi.nlm.nih.gov/pubmed/10801971

5 Srivastava R, Peterson MS, Bentley WE. Stochastic Kinetic Analysis of the Escherichia coli Stress Circuit Using 32 -Targeted Antisense. Biotechnol Bioeng [Internet]. 2001; 75:120-9. Available from: http://dept-info.labri. fr/ beurton/Enseignement/Modelisation/Articles/Sigma32Srivastava.pdf

6 Gamer J, Bujard H, Bukau B. Physical interaction between heat shock proteins DnaK, DnaJ, and GrpE and the bacterial heat shock transcription factor sigma 32. Cell [Internet]. 1992 May 29; 69(5):833-42. Available from: http:// 
7 Wang QP, Kaguni JM. A novel sigma factor is involved in expression of the rpoH gene of Escherichia coli. J Bacteriol [Internet]. American Society for Microbiology (ASM); 1989 Aug; 171(8):4248-53. Available from: http:// www.ncbi.nlm.nih.gov/pubmed/2546916

8 Kamath-Loeb AS, Gross CA. Translational regulation of sigma 32 synthesis: requirement for an internal control element. J Bacteriol [Internet]. 1991 Jun; 173(12):3904-6. Available from: http://www.ncbi.nlm.nih.gov/ pubmed/2050641

9 Nagai H, Yuzawa H, Yura T. Interplay of two cis-acting mRNA regions in translational control of sigma 32 synthesis during the heat shock response of Escherichia coli. Proc Natl Acad Sci U S A [Internet]. 1991 Dec 1; 88(23):10515-9. Available from: http://www.ncbi.nlm.nih.gov/pub$\mathrm{med} / 1961716$

10 Lim B, Miyazaki R, Neher S, Siegele DA, Ito K, Walter P, et al. Heat Shock Transcription Factor $\sigma 32$ Co-opts the Signal Recognition Particle to Regulate Protein Homeostasis in E. coli. Horwich AL, editor. PLoS Biol [Internet]. 2013 Dec 17; 11(12):e1001735. Available from: http://www.ncbi.nlm.nih. gov/pubmed/24358019

11 Grossman AD, Erickson JW, Gross CA. The htpR gene product of E. coli is a sigma factor for heat-shock promoters. Cell [Internet]. Elsevier; 1984 Sep 1; 38(2):383-90. Available from: http://www.ncbi.nlm.nih.gov/pub$\mathrm{med} / 6380765$

12 Funahashi A, Matsuoka Y, Jouraku A, Kitano H, Kikuchi N. Celldesigner: A Modeling Tool for Biochemical Networks. Proceedings of the 2006 Winter Simulation Conference [Internet]. IEEE; 2006. p. 1707-12. Available from: http://ieeexplore.ieee.org/document/4117804/

13 Funahashi A, Matsuoka Y, Jouraku A, Morohashi M, Kikuchi N, Kitano H CellDesigner 3.5: A Versatile Modeling Tool for Biochemical Networks. Proc IEEE [Internet]. 2008 Aug; 96(8):1254-65. Available from: http://ieeexplore.ieee.org/document/4567412/

14 Hoops S, Sahle S, Gauges R, Lee C, Pahle J, Simus N, et al. COPASI--a COmplex PAthway SImulator. Bioinformatics [Internet]. 2006 Dec 15; 22(24):3067-74. Available from: http://www.ncbi.nlm.nih.gov/pubmed/17032683

\section{Material complementario}

1. Sistema de ecuaciones diferenciales.

function $\mathrm{dx}=$ model_sigma32(t,x)

global k1 k2 k3 k4 k5 k6 k7 k8 k9 k10 k11 k12

$\mathrm{dx}=\mathrm{zeros}(6,1) ; \%$ Vector Columna

$\mathrm{dx}(1)=\mathrm{k} 1-\mathrm{k} 2 * \mathrm{x}(1)-\mathrm{k} 3 * \mathrm{x}(1)$;

$\mathrm{dx}(2)=\mathrm{k} 3 * \mathrm{x}(1)+\mathrm{k} 5 * \mathrm{x}(3)+\mathrm{k} 8 * \mathrm{x}(3)-\mathrm{k} 4 * \mathrm{x}(2)+\mathrm{k} 11 * \mathrm{x}(6)-\mathrm{k} 10 * \mathrm{x}(2) * \mathrm{x}(4)+\mathrm{k} 6 * \mathrm{x}(3)-\mathrm{k} 12 * \mathrm{x}(5) * \mathrm{x}(6)$;

$\mathrm{dx}(3)=\mathrm{k} 4 * \mathrm{x}(2)-\mathrm{k} 5 * \mathrm{x}(3)-\mathrm{k} 6 * \mathrm{x}(3)-\mathrm{k} 8 * \mathrm{x}(3)$;

$\mathrm{dx}(4)=\mathrm{k} 6 * \mathrm{x}(3)-\mathrm{k} 7 * \mathrm{x}(5)-\mathrm{k} 12 * \mathrm{x}(5) * \mathrm{x}(6)$;

$\mathrm{dx}(5)=\mathrm{k} 11 * \mathrm{x}(6)+\mathrm{k} 8 * \mathrm{x}(3)+\mathrm{k} 12 * \mathrm{x}(5) * \mathrm{x}(6)-\mathrm{k} 10 * \mathrm{x}(4) * \mathrm{x}(2)-\mathrm{k} 9 * \mathrm{x}(4)$;

$\mathrm{dx}(6)=\mathrm{k} 10 * \mathrm{x}(2) * \mathrm{x}(4)-\mathrm{k} 11 * \mathrm{x}(6)-\mathrm{k} 12 * \mathrm{x}(6) * \mathrm{x}(5)$;

2. Solución del sistema de ecuaciones diferenciales ordinarias (ode45)

global k1 k2 k3 k4 k5 k6 k7 k8 k9 k10 k11 k12;

\%Unidades

$\begin{array}{ll}\mathrm{k} 1=1.4 \mathrm{e}-5 ; & \% 1 / \mathrm{s} \\ \mathrm{k} 2=1.4 \mathrm{e}-6 ; & \% 1 / \mathrm{s} \\ \mathrm{k} 3=0.007 ; & \% 1 / \mathrm{s} \\ \mathrm{k} 4=0.7 ; & \% 1 / \mathrm{s} \\ \mathrm{k} 5=0.13 ; & \% 1 / \mathrm{nm}{ }^{*} \mathrm{~s} \\ \mathrm{k} 6=4.41 \mathrm{e}-3 ; & \% 1 / \mathrm{nm}^{*} \mathrm{~s} \\ \mathrm{k} 7=7.40 \mathrm{e}-11 ; & \% 1 / \mathrm{s} \\ \mathrm{k} 8=4.41 \mathrm{e}-3 ; & \% 1 / \mathrm{nm}^{*} \mathrm{~s} \\ \mathrm{k} 9=6.40 \mathrm{e}-10 ; & \% 1 / \mathrm{s} \\ \mathrm{k} 10=3.27 \mathrm{e}-4 ; & \% 1 / \mathrm{nms}^{*} \mathrm{~s} \\ \mathrm{k} 11=4.4 \mathrm{e}-4 ; & \% 1 / \mathrm{s} \\ \mathrm{k} 12=1.28 \mathrm{e}-6 ; & \% 1 / \mathrm{nm}^{*} \mathrm{~s}\end{array}$

$\begin{array}{ll}\mathrm{A}=1 ; & \text { \%Sigma32gen } \\ \mathrm{D}=1 ; & \text { \%ftshgen } \\ \mathrm{F}=1 ; & \text { \%Jcomp-gen } \\ \mathrm{Bo}=6 ; & \text { \%Sigma32mRNA } \\ \mathrm{Co}=9 ; & \text { \%Sigma32 } \\ \mathrm{I}=1 ; & \text { E-Sigma32 }\end{array}$




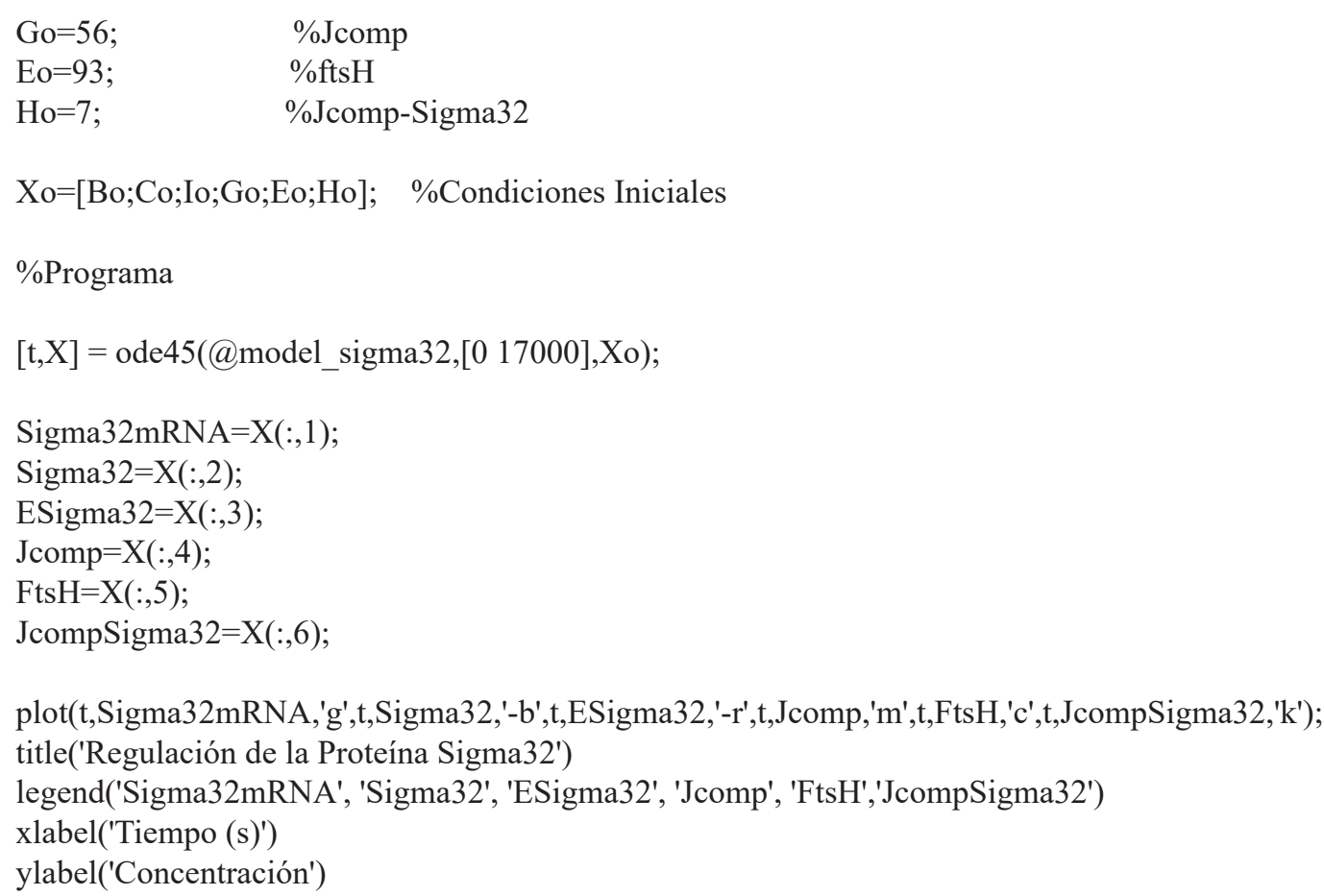

3. Análisis de sensibilidad.

clear all

$[\mathrm{t}, \mathrm{X}]=$ ode45(@model_sigma32,[0 80],Xo);

Sigma32mRNA=X(:,1);

Sigma32 $=\mathrm{X}(:, 2)$;

ESigma32 $=\mathrm{X}(:, 3)$;

Jcomp $=\mathrm{X}(:, 4)$;

$\mathrm{FtsH}=\mathrm{X}(:, 5)$;

JcompSigma32=X(:,6);

plot(t, Sigma32,'red');

hold on

$\mathrm{k} 8=4.41 \mathrm{e}-3 / 1000$;

for $\mathrm{i}=1: 5$

$\mathrm{k} 8=(\mathrm{k} 8 * 10)$;

$[\mathrm{t}, \mathrm{X}]=$ ode45(@model_sigma32,[0 60],Xo);

Sigma32mRNAv $=\mathrm{X}(:, 1)$;

Sigma32 $\mathrm{v}=\mathrm{X}(:, 2)$;

ESigma32 $v=X(:, 3)$;

Jcompv $=\mathrm{X}(:, 4)$;

$\mathrm{FtsHv}=\mathrm{X}(:, 5)$;

JcompSigma32v=X(:,6);

$\operatorname{plot}(\mathrm{t}$, Sigma32v,'black');

title('Cambio de la concentracion Sigma32 variando K8')

legend('Sigma32')

xlabel('Ti')

end 